\title{
BMC Psychiatry reviewer acknowledgement 2014
}

Catherine M Rice ${ }^{*}$ and Diana M Marshall

\section{Contributing reviewers}

The editors of BMC Psychiatry would like to thank all our reviewers who have contributed to the journal in Volume 14 (2014).

Eivind Aakhus

Norway

Giovanni Abbate-Daga

Italy

Chadi Abdallah

USA

Riadh Abed

UK

Marielle Abrahamse

Netherlands

Ashley Acheson

USA

Marcel Aebi

Switzerland

Brian Ahmedani

USA

Christopher Ahnallen

USA

Kirsi Ahola

Finland

Shahin Akhondzadeh

Iran

Chelsea Ale

USA

Shiri Altman

Israel

Paula Alves

Portugal
Benedikt Lorenz Amann
Spain

Bart Ament

Netherlands

Federico Amianto

Italy

Clare Anderson

Australia

Alessandro Angrilli

Italy

Jules Angst

Switzerland

Kurt Angstman

USA

Trevor Archer

Sweden

Iris Arends

Netherlands

Todd Arnedt

USA

Hakan Atalay

Turkey

Anna Rita Atti

Italy

Dana Atzil Slonim

Israel

Carlos Ayan

Spain
Sofie Bäärnhielm

Sweden

Enrique Baca-Garcia

Spain

Dusan Backovic

Serbia

Imke Baetens

Belgium

Amber Bahorik

USA

Gianluca Baio

UK

Maarten Bak

Netherlands

Amanda Baker

Australia

Eleanor Bantry White

Ireland

Yoram Barak

Israel

Mariapaola Barbato

Canada

Izabela Barbosa

Brazil

Nicola Barclay

UK

Rachel Barnes

USA

* Correspondence: Catherine.Rice@biomedcentral.com

BioMed Central, Floor 6, 236 Gray's Inn Road, London WC1X 8HB, UK 


\begin{tabular}{|c|c|c|}
\hline $\begin{array}{l}\text { Stefano Baroni } \\
\text { Italy }\end{array}$ & $\begin{array}{l}\text { Istvan Bitter } \\
\text { Hungary }\end{array}$ & $\begin{array}{l}\text { Joshua Breslau } \\
\text { USA }\end{array}$ \\
\hline $\begin{array}{l}\text { Giovanni Battista Bartolucci } \\
\text { Italy }\end{array}$ & $\begin{array}{l}\text { Simon Blackwell } \\
\text { UK }\end{array}$ & $\begin{array}{l}\text { Jeffrey Bridge } \\
\text { USA }\end{array}$ \\
\hline $\begin{array}{l}\text { Judith Bass } \\
\text { USA }\end{array}$ & $\begin{array}{l}\text { Sergio Blay } \\
\text { Brazil }\end{array}$ & $\begin{array}{l}\text { William Brinkman } \\
\text { USA }\end{array}$ \\
\hline $\begin{array}{l}\text { Deborah Bassett } \\
\text { USA }\end{array}$ & $\begin{array}{l}\text { Olof Blix } \\
\text { Sweden }\end{array}$ & $\begin{array}{l}\text { Teresita Briones } \\
\text { USA }\end{array}$ \\
\hline $\begin{array}{l}\text { Daniel Bateman } \\
\text { USA }\end{array}$ & $\begin{array}{l}\text { D Blumberger } \\
\text { Canada }\end{array}$ & $\begin{array}{l}\text { Markus Britschgi } \\
\text { Switzerland }\end{array}$ \\
\hline $\begin{array}{l}\text { Stephanie Bauer } \\
\text { Germany }\end{array}$ & $\begin{array}{l}\text { Sune Bo } \\
\text { Denmark }\end{array}$ & $\begin{array}{l}\text { June Brown } \\
\text { UK }\end{array}$ \\
\hline $\begin{array}{l}\text { Thomas Beblo } \\
\text { Germany }\end{array}$ & $\begin{array}{l}\text { Jed Boardman } \\
\text { UK }\end{array}$ & $\begin{array}{l}\text { Margaret Brown } \\
\text { Australia }\end{array}$ \\
\hline $\begin{array}{l}\text { Margherita Bechi } \\
\text { Italy }\end{array}$ & $\begin{array}{l}\text { Petr Bob } \\
\text { Czech Republic }\end{array}$ & $\begin{array}{l}\text { Martin Bruene } \\
\text { Germany }\end{array}$ \\
\hline $\begin{array}{l}\text { Arthur Becker-Weidman } \\
\text { USA }\end{array}$ & $\begin{array}{l}\text { Julio Bobes } \\
\text { Spain }\end{array}$ & $\begin{array}{l}\text { Scott Brunero } \\
\text { Australia }\end{array}$ \\
\hline $\begin{array}{l}\text { Hassan Bella } \\
\text { Saudi Arabia }\end{array}$ & $\begin{array}{l}\text { Iwona Bojar } \\
\text { Poland }\end{array}$ & $\begin{array}{l}\text { Andre Brunoni } \\
\text { Brazil }\end{array}$ \\
\hline $\begin{array}{l}\text { Martino Belvederi Murri } \\
\text { Italy }\end{array}$ & $\begin{array}{l}\text { Ilaria Bonoldi } \\
\text { UK }\end{array}$ & $\begin{array}{l}\text { Fabrizio Bruschi } \\
\text { Italy }\end{array}$ \\
\hline $\begin{array}{l}\text { Raoul Belzeaux } \\
\text { France }\end{array}$ & $\begin{array}{l}\text { Rhonda Booth } \\
\text { UK }\end{array}$ & $\begin{array}{l}\text { Kelly Buck } \\
\text { USA }\end{array}$ \\
\hline $\begin{array}{l}\text { Susan Mary Benbow } \\
\text { UK }\end{array}$ & $\begin{array}{l}\text { Rami Bou Khalil } \\
\text { France }\end{array}$ & $\begin{array}{l}\text { Kyle Burghardt } \\
\text { USA }\end{array}$ \\
\hline $\begin{array}{l}\text { Maneeton Benchalak } \\
\text { Thailand }\end{array}$ & $\begin{array}{l}\text { Aurore Boulard } \\
\text { Belgium }\end{array}$ & $\begin{array}{l}\text { Julius Burkauskas } \\
\text { Lithuania }\end{array}$ \\
\hline $\begin{array}{l}\text { Kristina Bennert } \\
\text { UK }\end{array}$ & $\begin{array}{l}\text { Jennifer Boyd } \\
\text { USA }\end{array}$ & $\begin{array}{l}\text { Lisa Burke } \\
\text { Australia }\end{array}$ \\
\hline $\begin{array}{l}\text { Dror Ben-Zeev } \\
\text { USA }\end{array}$ & $\begin{array}{l}\text { Laurent Boyer } \\
\text { France }\end{array}$ & $\begin{array}{l}\text { Christopher Bushe } \\
\text { UK }\end{array}$ \\
\hline $\begin{array}{l}\text { Yemane Berhane } \\
\text { Ethiopia }\end{array}$ & $\begin{array}{l}\text { Lindy-Lou Boyette } \\
\text { Netherlands }\end{array}$ & $\begin{array}{l}\text { Andrew Bushmakin } \\
\text { USA }\end{array}$ \\
\hline $\begin{array}{l}\text { Kalpana Bhaskaran } \\
\text { Singapore }\end{array}$ & $\begin{array}{l}\text { Abby Braden } \\
\text { USA }\end{array}$ & $\begin{array}{l}\text { Casimiro Cabrera Abreu } \\
\text { Canada }\end{array}$ \\
\hline $\begin{array}{l}\text { Kamaldeep Bhui } \\
\text { UK }\end{array}$ & $\begin{array}{l}\text { Joanne Brady } \\
\text { USA }\end{array}$ & $\begin{array}{l}\text { Raffaele Cacciaglia } \\
\text { Spain }\end{array}$ \\
\hline $\begin{array}{l}\text { Andargachew Kassa Biratu } \\
\text { Ethiopia }\end{array}$ & $\begin{array}{l}\text { Nicholas Breitborde } \\
\text { USA }\end{array}$ & $\begin{array}{l}\text { John Cacioppo } \\
\text { USA }\end{array}$ \\
\hline $\begin{array}{l}\text { Victoria Bird } \\
\text { UK }\end{array}$ & $\begin{array}{l}\text { Benjamin Brent } \\
\text { USA }\end{array}$ & $\begin{array}{l}\text { Kristin Cadenhead } \\
\text { USA }\end{array}$ \\
\hline $\begin{array}{l}\text { Carl Birmingham } \\
\text { Canada }\end{array}$ & $\begin{array}{l}\text { David Brent } \\
\text { USA }\end{array}$ & $\begin{array}{l}\text { Joseph Calabrese } \\
\text { USA }\end{array}$ \\
\hline
\end{tabular}




\begin{tabular}{|c|c|c|}
\hline Alison Calear & Manoranjenni Chetty & Samuele Cortese \\
\hline Australia & UK & USA \\
\hline Michael Caligiuri & Dixon Chibanda & William Coryell \\
\hline USA & Zimbabwe & USA \\
\hline John Cape & Howard Chilcoat & Philippe Courtet \\
\hline UK & USA & France \\
\hline Alejandra Caqueo-Urizar & Weng Yee Chin & Kay Lorraine Cox \\
\hline Chile & Hong Kong & Australia \\
\hline R. Nicholas Carleton & Dan Chisholm & Paul Crits-Christoph \\
\hline Canada & Switzerland & USA \\
\hline Hannah Carliner & Pratap Chokka & Anselm Crombach \\
\hline USA & Canada & Germany \\
\hline Vaughan Carr & Yuan-Hwa Chou & Gary Cuddeback \\
\hline Australia & Taiwan & USA \\
\hline Giovanni Castellini & David Christmas & Alexis Cullen \\
\hline Italy & UK & UK \\
\hline Carl Castro & Quetzal Class & Kathryn Cullen \\
\hline USA & USA & USA \\
\hline Ferrán Catalá-López & Claude Robert Cloninger & Larry Culpepper \\
\hline Spain & USA & USA \\
\hline Pascal Cathebras & Laurie Clune & Caroline Dalton \\
\hline France & Canada & UK \\
\hline Hilal Ceit & Vanessa Cobham & Gerhard Dammann \\
\hline Netherlands & Australia & Switzerland \\
\hline Eduardo Chachamovich & Alex Cohen & Louise Danielsson \\
\hline Canada & UK & Sweden \\
\hline Subhajit Chakravorty & Moran Cohn & Alison Darcy \\
\hline USA & Netherlands & USA \\
\hline Lai Fong Chan & Hannie Comijs & Carl D'Arcy \\
\hline Malaysia & Netherlands & Canada \\
\hline Raymond Chan & David Conn & Renaud David \\
\hline China & Canada & France \\
\hline Kristine Chapleau & Amy Conrad & Larry Davidson \\
\hline USA & USA & USA \\
\hline Robert Chaplin & Andrew Cook & Darlene Davis \\
\hline UK & USA & USA \\
\hline Gregory Chasson & Maurizio Coppola & Derek De Beurs \\
\hline USA & Italy & Netherlands \\
\hline Imran Chaudhry & Rhiannon Corcoran & Nicola De Carlo \\
\hline UK & UK & Italy \\
\hline Huafu Chen & Angel Correa & Giovanni De Girolamo \\
\hline China & Spain & Italy \\
\hline Chih-Ying Chen & Deborah Corring & Peter De Jonge \\
\hline USA & Canada & Netherlands \\
\hline
\end{tabular}


Flora De La Barra

Chile

Jose De Leon

USA

Jesus De Pedro-Cuesta

Spain

Varuni De Silva

Sri Lanka

Nicholas Deakin

UK

Catherine Deeprose

UK

Ryan Delapp

USA

Philippe Delespaul

Netherlands

Chao Deng

Australia

Joseph Deng

USA

Niklaus Denier

Switzerland

Birgit Derntl

Germany

Alain Dervaux

France

David Diamond

USA

Albert Diefenbacher

Germany

Danai Dima

UK

Steven Dobscha

USA

Tara Donker

Australia

Sarah Doucette

Canada

Darin Dougherty

USA

Irena Draskovic

Netherlands

Boris Drozdek

Netherlands
Fei Du

USA

Ke-Lin Du

China

Arnaud Duhoux

Canada

Sophie Duranceau

Canada

Nicola Dusi

Italy

John Dziak

USA

Bjorn Ebdrup

Denmark

Lara Ebenfeld

Germany

David Daniel Ebert

Germany

Chad Ebesutani

Korea, South

Takashi Ebisawa

Japan

Thomas Ehring

Germany

Solvig Ekblad

Sweden

Thomas Elbert

Germany

Jennifer Elliott

USA

Graham Emslie

USA

Jerome Endrass

Switzerland

Harald Engler

Germany

Linda Ercoli

USA

Pascale Esch

Luxembourg

Ozlem Eylem

Netherlands

Harris Eyre

Australia
Sonya Faber

Germany

Xiang Fan

USA

Xiaoduo Fan

USA

Aida Farreny

Spain

Ken Farrington

UK

Secondo Fassino

Italy

Johannes Fellinger

Austria

Liang Feng

Singapore

Christopher Ferguson

USA

Jilles Fermont

UK

Ana Fernandez

Spain

Maria Isabel Fernández San Martín

Spain

Fernando Fernandez-Aranda

Spain

Mark Ferro

Canada

Mathew Fetzner

Canada

Jess Fiedorowicz

USA

Andrea Fiorillo

Italy

Sarah Fischer

USA

Ellen Fitzsimmons-Craft

USA

Amos Fleischmann

Israel

Marie-Josee Fleury

Canada

Leon Flicker

Australia 


\begin{tabular}{|c|c|c|}
\hline $\begin{array}{l}\text { Tim Fong } \\
\text { USA }\end{array}$ & $\begin{array}{l}\text { Fiona Gaughran } \\
\text { UK }\end{array}$ & $\begin{array}{l}\text { Carlos Gois } \\
\text { Portugal }\end{array}$ \\
\hline $\begin{array}{l}\text { Eduardo Fonseca Pedrero } \\
\text { Spain }\end{array}$ & $\begin{array}{l}\text { Caterina Gawrilow } \\
\text { Germany }\end{array}$ & $\begin{array}{l}\text { Lutz Goldbeck } \\
\text { Germany }\end{array}$ \\
\hline $\begin{array}{l}\text { David Forbes } \\
\text { Australia }\end{array}$ & $\begin{array}{l}\text { Measho Gebregziabher } \\
\text { Ethiopia }\end{array}$ & $\begin{array}{l}\text { David Goldberg } \\
\text { UK }\end{array}$ \\
\hline $\begin{array}{l}\text { Thomas Forkmann } \\
\text { Germany }\end{array}$ & $\begin{array}{l}\text { James Gedra } \\
\text { USA }\end{array}$ & $\begin{array}{l}\text { Hadass Goldblatt } \\
\text { Israel }\end{array}$ \\
\hline $\begin{array}{l}\text { Tomislav Franic } \\
\text { Croatia }\end{array}$ & $\begin{array}{l}\text { Bizu Gelaye } \\
\text { USA }\end{array}$ & $\begin{array}{l}\text { Andrea Goldschmidt } \\
\text { USA }\end{array}$ \\
\hline $\begin{array}{l}\text { Kenneth Freedland } \\
\text { USA }\end{array}$ & $\begin{array}{l}\text { Dejan Georgiev } \\
\text { UK }\end{array}$ & $\begin{array}{l}\text { Jesus Gomar } \\
\text { USA }\end{array}$ \\
\hline $\begin{array}{l}\text { Richard Frye } \\
\text { USA }\end{array}$ & $\begin{array}{l}\text { Adam Gerace } \\
\text { Australia }\end{array}$ & $\begin{array}{l}\text { Marcus Gomes } \\
\text { Brazil }\end{array}$ \\
\hline $\begin{array}{l}\text { Sanae Fukuda } \\
\text { Japan }\end{array}$ & $\begin{array}{l}\text { Adam Geraghty } \\
\text { UK }\end{array}$ & $\begin{array}{l}\text { Rafael Gonzalez } \\
\text { UK }\end{array}$ \\
\hline $\begin{array}{l}\text { Sadaaki Fukui } \\
\text { USA }\end{array}$ & $\begin{array}{l}\text { Lilian Ghandour } \\
\text { Lebanon }\end{array}$ & $\begin{array}{l}\text { Robert Gonzalez } \\
\text { USA }\end{array}$ \\
\hline $\begin{array}{l}\text { Daniel Fung } \\
\text { Singapore }\end{array}$ & $\begin{array}{l}\text { Lucio Ghio } \\
\text { Italy }\end{array}$ & $\begin{array}{l}\text { Alexandre Gonzalez-Rodriguez } \\
\text { Spain }\end{array}$ \\
\hline $\begin{array}{l}\text { Adel Gabriel } \\
\text { Canada }\end{array}$ & $\begin{array}{l}\text { Domenico Giacco } \\
\text { UK }\end{array}$ & $\begin{array}{l}\text { Peter Goossens } \\
\text { Netherlands }\end{array}$ \\
\hline $\begin{array}{l}\text { Patrick Gajewski } \\
\text { Germany }\end{array}$ & $\begin{array}{l}\text { Katrin Giel } \\
\text { Germany }\end{array}$ & $\begin{array}{l}\text { Jessica Goren } \\
\text { USA }\end{array}$ \\
\hline $\begin{array}{l}\text { Gilad Gal } \\
\text { Israel }\end{array}$ & $\begin{array}{l}\text { Itzhak Gilat } \\
\text { Israel }\end{array}$ & $\begin{array}{l}\text { Carla Maria Gramaglia } \\
\text { Italy }\end{array}$ \\
\hline $\begin{array}{l}\text { Silvia Gallagher } \\
\text { Ireland }\end{array}$ & $\begin{array}{l}\text { Stephen Gilman } \\
\text { USA }\end{array}$ & $\begin{array}{l}\text { Iria Grande } \\
\text { Spain }\end{array}$ \\
\hline $\begin{array}{l}\text { Juan Gallego } \\
\text { USA }\end{array}$ & $\begin{array}{l}\text { Damiano Girardi } \\
\text { Italy }\end{array}$ & $\begin{array}{l}\text { Michael Grandner } \\
\text { USA }\end{array}$ \\
\hline $\begin{array}{l}\text { Orsola Gambini } \\
\text { Italy }\end{array}$ & $\begin{array}{l}\text { Ragy Girgis } \\
\text { USA }\end{array}$ & $\begin{array}{l}\text { Kelly Green } \\
\text { USA }\end{array}$ \\
\hline $\begin{array}{l}\text { Matthew Gambino } \\
\text { USA }\end{array}$ & $\begin{array}{l}\text { Lydia Gisle } \\
\text { Belgium }\end{array}$ & $\begin{array}{l}\text { Tracy Greer } \\
\text { USA }\end{array}$ \\
\hline $\begin{array}{l}\text { David Garcia } \\
\text { Switzerland }\end{array}$ & $\begin{array}{l}\text { Melissa Gladstone } \\
\text { UK }\end{array}$ & $\begin{array}{l}\text { Sebastien Grenier } \\
\text { Canada }\end{array}$ \\
\hline $\begin{array}{l}\text { Ricardo García-Mayor } \\
\text { Spain }\end{array}$ & $\begin{array}{l}\text { Heide Glaesmer } \\
\text { Germany }\end{array}$ & $\begin{array}{l}\text { James Griffith } \\
\text { USA }\end{array}$ \\
\hline $\begin{array}{l}\text { Genevieve Gariepy } \\
\text { Canada }\end{array}$ & $\begin{array}{l}\text { Joseph Glass } \\
\text { USA }\end{array}$ & $\begin{array}{l}\text { Tamasine Grimes } \\
\text { Ireland }\end{array}$ \\
\hline $\begin{array}{l}\text { Andrew Garratt } \\
\text { Norway }\end{array}$ & $\begin{array}{l}\text { Robert Göder } \\
\text { Germany }\end{array}$ & $\begin{array}{l}\text { Sandeep Grover } \\
\text { India }\end{array}$ \\
\hline $\begin{array}{l}\text { Faye Gary } \\
\text { USA }\end{array}$ & $\begin{array}{l}\text { Betty Goguikian Ratcliff } \\
\text { Switzerland }\end{array}$ & $\begin{array}{l}\text { Anne Grundy } \\
\text { Canada }\end{array}$ \\
\hline
\end{tabular}




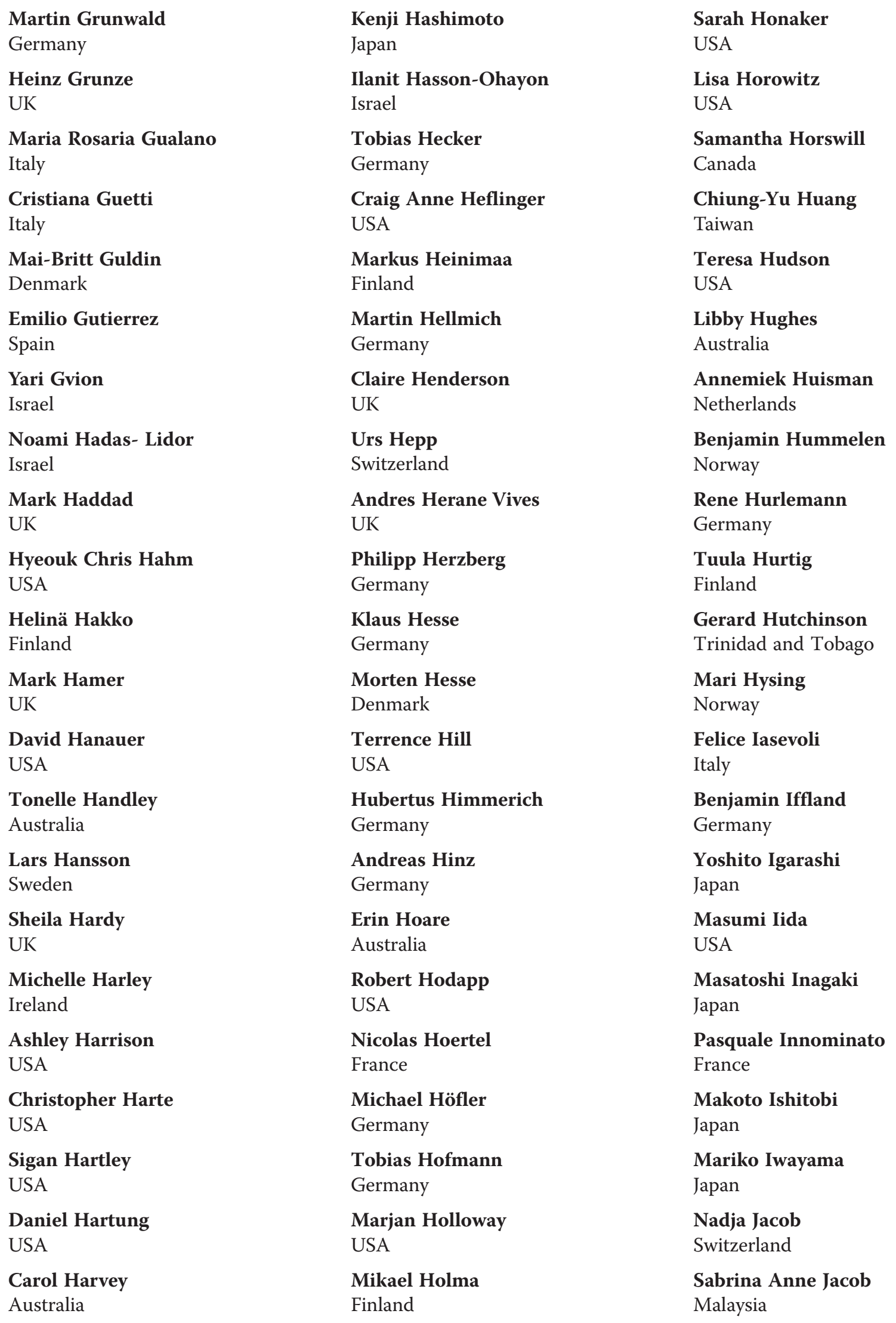


Frank Jacobi

Germany

Susanne Jaeger

Germany

Anthony James

UK

Josee L Jarry

Canada

Rachel Jenkins

UK

Tawanchai Jirapramukpitak

Thailand

Stefan Johansson

Norway

Bjorn Johnson

Sweden

Mandy Johnstone

UK

Sheila Jones

Denmark

Ruud Jongedijk

Netherlands

Andreas Joos

Germany

Rikke Jørgensen

Denmark

Georg Juckel

Germany

Minyoung Jung

Korea, South

Mario Juruena

Brazil

Colette Kabrita

Lebanon

Michael Kaess

Germany

Kirsti Kähärä

Finland

Ondrej Kalina

Slovakia

Bengt Källén

Sweden

Eva Kaltenthaler

UK
Inge Kamp-Becker

Germany

Olli Kampman

Finland

Richard Antony Alexander Kanaan

UK

Yukiko Kano

Japan

Joshua Kantrowitz

USA

Manav Kapoor

USA

Nestor Damian Kapusta

Austria

Nilamadhab Kar

UK

Susanne Karch

Bosnia and Herzegovina

Andrea Kass

USA

Takahiro Kato

Japan

Marcia Kauer-Sant'Anna

Brazil

Brooke Kauffman

USA

Anne Kavanagh

Australia

Yoshitaka Kawashima

Japan

Robert Keeley

USA

Matthew Keller

USA

Friederike Kendel

Germany

Harry Kennedy

Ireland

Martin Kennedy

New Zealand

Michelle Kermode

Australia

Sarah Kertz

USA
Alice Keski-Valkama

Finland

Ronald Kessler

USA

Hari-Mandir Khalsa

USA

Judi Kidger

UK

Jae-Min Kim

Korea, South

Yong Sik Kim

Korea, South

David Kingdon

UK

John David Kinzie

USA

Julia Kirkham

Canada

Anne Kleinsasser

USA

Elizabeth Klerman

USA

Sören Kliem

Germany

Stefan Kloiber

Germany

Christine Knaevelsrud

Germany

Jeroen Knipscheer

Netherlands

Sarah Knowles

UK

Kathrin Koch

Germany

Manami Kodaka

Japan

Stephan Köhler

Germany

Michael Kohn

Australia

Tsuyoshi Kondo

Japan

Barna Konkoly Thege

Canada 


\begin{tabular}{|c|c|c|}
\hline $\begin{array}{l}\text { Dimitrios Kontis } \\
\text { Greece }\end{array}$ & $\begin{array}{l}\text { Brian Lakey } \\
\text { USA }\end{array}$ & $\begin{array}{l}\text { Jean-Pierre Lindenmayer } \\
\text { USA }\end{array}$ \\
\hline $\begin{array}{l}\text { Hans Koppeschaar } \\
\text { Netherlands }\end{array}$ & $\begin{array}{l}\text { Marie-Eve Lamontagne } \\
\text { Canada }\end{array}$ & $\begin{array}{l}\text { Jamey Lister } \\
\text { USA }\end{array}$ \\
\hline $\begin{array}{l}\text { Jyrki Korkeila } \\
\text { Finland }\end{array}$ & $\begin{array}{l}\text { Carol Landis } \\
\text { USA }\end{array}$ & $\begin{array}{l}\text { Xianhua Liu } \\
\text { China }\end{array}$ \\
\hline $\begin{array}{l}\text { Hirotaka Kosaka } \\
\text { Japan }\end{array}$ & $\begin{array}{l}\text { Nils Inge Landrfø } \\
\text { Norway }\end{array}$ & $\begin{array}{l}\text { Zhongchun Liu } \\
\text { China }\end{array}$ \\
\hline $\begin{array}{l}\text { Ernst Koster } \\
\text { Belgium }\end{array}$ & $\begin{array}{l}\text { Pierre Lau } \\
\text { Belgium }\end{array}$ & $\begin{array}{l}\text { James Livingston } \\
\text { Canada }\end{array}$ \\
\hline $\begin{array}{l}\text { Diana Koszycki } \\
\text { Canada }\end{array}$ & $\begin{array}{l}\text { Chi-Kin Law } \\
\text { Hong Kong }\end{array}$ & $\begin{array}{l}\text { Brynmor Lloyd-Evans } \\
\text { UK }\end{array}$ \\
\hline $\begin{array}{l}\text { Leda Kovatsi } \\
\text { Greece }\end{array}$ & $\begin{array}{l}\text { Robyne Le Brocque } \\
\text { Australia }\end{array}$ & $\begin{array}{l}\text { Alexandre Loch } \\
\text { Brazil }\end{array}$ \\
\hline $\begin{array}{l}\text { Kate Krajci } \\
\text { USA }\end{array}$ & $\begin{array}{l}\text { Monique Lebourgeois } \\
\text { USA }\end{array}$ & $\begin{array}{l}\text { Steven Lockley } \\
\text { USA }\end{array}$ \\
\hline $\begin{array}{l}\text { Ueli Kramer } \\
\text { Switzerland }\end{array}$ & $\begin{array}{l}\text { Daniel Lebouthillier } \\
\text { Canada }\end{array}$ & $\begin{array}{l}\text { Paul Lombroso } \\
\text { USA }\end{array}$ \\
\hline $\begin{array}{l}\text { Levente Kriston } \\
\text { Germany }\end{array}$ & $\begin{array}{l}\text { David Ledgerwood } \\
\text { USA }\end{array}$ & $\begin{array}{l}\text { Barbara Lopes Cardozo } \\
\text { USA }\end{array}$ \\
\hline $\begin{array}{l}\text { Kurt Kroenke } \\
\text { USA }\end{array}$ & $\begin{array}{l}\text { William Lee } \\
\text { UK }\end{array}$ & $\begin{array}{l}\text { Jorge Lopez-Castroman } \\
\text { Spain }\end{array}$ \\
\hline $\begin{array}{l}\text { Jesper Krogh } \\
\text { Denmark }\end{array}$ & $\begin{array}{l}\text { Robert Leeman } \\
\text { USA }\end{array}$ & $\begin{array}{l}\text { Lee-Fay Low } \\
\text { Australia }\end{array}$ \\
\hline $\begin{array}{l}\text { Ziad Kronfol } \\
\text { Qatar }\end{array}$ & $\begin{array}{l}\text { Seblewengel Lemma } \\
\text { Ethiopia }\end{array}$ & $\begin{array}{l}\text { Evan Lutkenhoff } \\
\text { USA }\end{array}$ \\
\hline $\begin{array}{l}\text { Hans Kroon } \\
\text { Netherlands }\end{array}$ & $\begin{array}{l}\text { Bethany Leonhardt } \\
\text { USA }\end{array}$ & $\begin{array}{l}\text { Sean Lynch } \\
\text { UK }\end{array}$ \\
\hline $\begin{array}{l}\text { Tillmann Kruger } \\
\text { Germany }\end{array}$ & $\begin{array}{l}\text { Karolina Leopold } \\
\text { Germany }\end{array}$ & $\begin{array}{l}\text { John Lyne } \\
\text { Ireland }\end{array}$ \\
\hline $\begin{array}{l}\text { Robert Kumsta } \\
\text { Afghanistan }\end{array}$ & $\begin{array}{l}\text { Stefan Leucht } \\
\text { Germany }\end{array}$ & $\begin{array}{l}\text { Corey Mackenzie } \\
\text { Canada }\end{array}$ \\
\hline $\begin{array}{l}\text { Matthew Kurtz } \\
\text { USA }\end{array}$ & $\begin{array}{l}\text { Catharine J. Lewis } \\
\text { Germany }\end{array}$ & $\begin{array}{l}\text { Helen Mactier } \\
\text { UK }\end{array}$ \\
\hline $\begin{array}{l}\text { Philipp Kuwert } \\
\text { Germany }\end{array}$ & $\begin{array}{l}\text { Jun Li } \\
\text { China }\end{array}$ & $\begin{array}{l}\text { Franziska Maier } \\
\text { Germany }\end{array}$ \\
\hline $\begin{array}{l}\text { Jeoung A Kwon } \\
\text { Korea, South }\end{array}$ & $\begin{array}{l}\text { Kaigang Li } \\
\text { USA }\end{array}$ & $\begin{array}{l}\text { Thomas Maier } \\
\text { Switzerland }\end{array}$ \\
\hline $\begin{array}{l}\text { Cornbelis Laban } \\
\text { Netherlands }\end{array}$ & $\begin{array}{l}\text { Pesach Lichtenberg } \\
\text { Israel }\end{array}$ & $\begin{array}{l}\text { Pallab Majumder } \\
\text { UK }\end{array}$ \\
\hline $\begin{array}{l}\text { Jean Lachaine } \\
\text { Canada }\end{array}$ & $\begin{array}{l}\text { Belinda Liddell } \\
\text { Australia }\end{array}$ & $\begin{array}{l}\text { Marelign Malaju } \\
\text { Ethiopia }\end{array}$ \\
\hline $\begin{array}{l}\text { Daniel Lai } \\
\text { Canada }\end{array}$ & $\begin{array}{l}\text { Shih-Ku Lin } \\
\text { Taiwan }\end{array}$ & $\begin{array}{l}\text { Sumaya Mall } \\
\text { USA }\end{array}$ \\
\hline
\end{tabular}


Jerome Maller

Australia

Stefania Mannarini

Italy

Donatella Marazziti

Italy

Angelo Giovanni Icro Maremmani Italy

Dominik Marin

Germany

Michel Maron

France

Colin Martin

UK

Leticia Martínez González

Spain

Jose Martinez-Raga

Spain

Francesca Martino

Italy

Matteo Martino

Italy

Enrica Marzola

Italy

Susan Marzolini

Canada

Holly Mash

USA

Julie Maslowsky

USA

Davide Massidda

Italy

Aleksandra Matanov

UK

Faith Matcham

UK

Catherine Mathews

South Africa

Yuki Matsumoto

Japan

Fermin Mayoral

Spain

Patrick Mccabe

USA
Rosemarie Mccabe

UK

Terence Mccann

Australia

Kevin Mccarthy

USA

Troy Mcewan

Australia

Gary Mclean

UK

Hamish Mcleod

UK

Caitlin Mcomish

Australia

Melissa Mcpheeters

USA

Nick Meader

UK

Denis Meadows

Australia

Ybe Meesters

Netherlands

Alison Menatti

USA

Itiana Castro Menezes

Brazil

Andreas Menke

Germany

Fiona Mensah

UK

Lisa Merlo

USA

Thomas Messer

Germany

Ricarda Mewes

Germany

Patrick Michaels

USA

Makoto Michikawa

Japan

Daniela Mier

Germany

Jouko Miettunen

Finland
Rafael T Mikolajczyk

Germany

Brian Miller

USA

Alessandra Minelli

Italy

Silvia Minozzi

Italy

Regina Miranda

USA

Mansha Mirza

USA

Mitsuhiro Miyashita

Japan

Toshiki Mizuno

Japan

Shakeh Moamrtin

Australia

Sara Modig

Sweden

Roy Moncayo

Austria

Akira Monji

Japan

Christiane Montag

Germany

Ali Montazeri

Iran

Galia Moran

Israel

Carlos Renato Moreira Maia

Brazil

Kevin Morgan

UK

Elaine Morrato

USA

Richard Morriss

UK

Anett Mueller-Alcazar

Germany

Gerry Mugford

Canada

Amin Muhammad Gadit

Canada 
Matthias J Müller

Germany

Astrid Müller

Germany

Gioia Mura

Italy

Stuart Murray

Australia

Jochen Mutschler

Switzerland

Maria Muzik

USA

Inez Myin-Germeys

Netherlands

Makalani Myrtveit

Norway

Michael Nadorff

USA

Farooq Naeem

Pakistan

Akiko Nakagawa

Japan

Takashi Nakamae

Japan

Hideyuki Nakane

Japan

Ora Nakash

Israel

Kristin Naragon-Gainey

USA

Davide Nardo

Italy

Carryl Navalta

USA

Manuela Neuman

Canada

Kelly Newell

Australia

James Newham

UK

Christian Hans Nickel

Switzerland

Angela Nickerson

USA
Inga Niedtfeld

Germany

Dana Jh Niehaus

South Africa

Katsuji Nishimura

Japan

Maria Nobile

Italy

Egil Nygaard

Norway

Shwe Zin Nyunt

Singapore

Michael Obermeier

Germany

Louise O'Brien

USA

Daniel O'Connor

Australia

Bridianne O'Dea

Australia

Michael Gerhard Odenwald

Germany

Brian O'Donoghue

Australia

Jeneva Ohan

Australia

Takashi Okada

Japan

Yasuyuki Okumura

Japan

Francesco Oliva

Italy

Javier Oltra-Cucarella

Spain

Joyce O'Mahony

Canada

Sepideh Omidvari

Iran

Juliana Onwumere

UK

Claire O'Reilly

Australia

Vasiliki Orgeta

UK
Killian O'Rourke

Ireland

Maciej Owecki

Poland

Shane Owens

USA

Yuji Ozeki

Japan

Seline Ozer

UK

Chi-Un Pae

Korea, South

Andrew Page

Australia

Daniel Pagnin

Brazil

Utpal Pajvani

USA

Ståle Pallesen

Norway

Maria Panagioti

UK

Ananda Pandurangi

USA

Igor Pantic

Serbia

A-La Park

UK

Sohee Park

USA

Barbara Parry

USA

Timo Partonen

Finland

Augusto Pasini

Italy

Sue Patterson

Australia

Lenin Pavon

Mexico

Nalin Payakachat

USA

Martha Payne

USA 
Carsten Bøcker Pedersen

Denmark

Kirsi Peltonen

France

Cinzia Perlini

Italy

Jane Persons

USA

Inge Petersen

South Africa

Anett Pfeiffer

Uganda

Andrea Pfennig

Germany

Ruth Pidsley

Australia

Anilkumar Pillai

USA

Martin Pinquart

Germany

Melissa Pinto

USA

Yehuda Pollak

Israel

Nunzio Pomara

USA

Maurizio Pompili

Italy

Alexander Ponizovsky

Israel

Piero Porcelli

Italy

Maj-Britt Posserud

Norway

Robert Powers

USA

Sarah Pratt

USA

Peter Pregelj

Slovenia

Antonio Preti

Italy

Stefan Priebe

UK
Petroula Proitsi

UK

David Pulsford

UK

Stephen Puntis

UK

Marianna Purgato

Italy

Bernd Puschner

Germany

Eli Puterman

USA

Terry Quinn

UK

Daniel Racey

UK

Mikael Rahmqvist

Sweden

Mahesh Rajasuriya

Sri Lanka

Anto P. Rajkumar

Denmark

Padmavati Ramachandran India

Hugh Ramsay

Ireland

Beverley Raphael

Australia

Carla Rash

USA

Silvia Ravera

Netherlands

Ursula Read

UK

Nicola Reavley

Australia

Elliott Rees

UK

Susan Rees

Australia

Gayle Reiber

USA

Kathryn Reid

USA
Lucia Reisch

Denmark

Javier Rejas

Spain

Solomon Renati

India

Leslie Rescorla

USA

Petra Retz-Junginger

Germany

Valdo Ricca

Italy

Derek Richards

Ireland

Debra Rickwood

Australia

Philipp S. Ritter

Germany

Patricio Riva-Posse

USA

Brandy Roane

USA

Laurence Inès Martine Robel

France

Pasquale Roberge

Canada

David Roberts

USA

Seren Roberts

UK

Jo Robinson

Australia

Dan Robotham

UK

James Roerig

USA

Paul Rohde

USA

Suely Roizenblatt

Brazil

Maria Graciela Rojas Castillo

Chile

Martha Romero

Mexico 


\begin{tabular}{|c|c|c|}
\hline Roberto Rona & Andrew Ryder & Marc Schmid \\
\hline UK & Canada & Switzerland \\
\hline Kathlyn Ronaldson & Ad Sad & Regien Schoemaker \\
\hline Australia & Bahrain & Netherlands \\
\hline Rita Roncone & Hans Inge Sævareid & Georg Schomerus \\
\hline Italy & Norway & Germany \\
\hline Jennifer Rose & Yoichi Sakakihara & Daniel Schöttle \\
\hline USA & Japan & Germany \\
\hline Roderick Rose & Joao Salgado & Beate M. Schulze \\
\hline USA & Brazil & Switzerland \\
\hline Irwin Rosenfarb & Raimo K. R. Salokangas & Jan Scott \\
\hline USA & Finland & UK \\
\hline Daniel Rossignol & Harshal Salve & Bridie Scott-Parker \\
\hline USA & India & Bahamas \\
\hline Francesco Rotella & Virginio Salvi & Dallas Seitz \\
\hline Italy & Italy & Canada \\
\hline Cecile Rousseau & Zainab Samaan & Carmen Senra \\
\hline Canada & Canada & Spain \\
\hline Guy Rousseau & Stephen Sammut & Michael Sernyak \\
\hline Canada & USA & USA \\
\hline Michael Roy & Marsal Sanches & Argentina Servin \\
\hline USA & USA & USA \\
\hline Abraham Rudnick & Marco Sarchiapone & Emanuel Severus \\
\hline Canada & Italy & Germany \\
\hline Montserrat Rue & Jerome Sarris & Asim Shah \\
\hline Spain & Australia & USA \\
\hline Reiner Rugulies & Tsuyoshi Sasaki & Katherine Sharkey \\
\hline Denmark & Japan & USA \\
\hline M Angeles Ruiperez & Anna Sasdelli & Ann Sharpley \\
\hline Spain & Italy & UK \\
\hline Miguel Ruiz-Veguilla & Cathrin Sauer & Taiwo Lateef Sheikh \\
\hline Spain & Germany & Nigeria \\
\hline Dan Rujescu & Broderick Sawyer & Yukihiko Shirayama \\
\hline Germany & USA & Japan \\
\hline Zlatka Russinova & Andrea Sboner & Dvora Shmulewitz \\
\hline USA & USA & USA \\
\hline Jennifer Rusted & Aart Schene & Michael Shuman \\
\hline UK & Netherlands & USA \\
\hline Torleif Ruud & Jeffrey Scherrer & Tianmei Si \\
\hline Norway & USA & China \\
\hline Joanne Ryan & Robert Schlack & Etienne Sibille \\
\hline Australia & Germany & USA \\
\hline Janusz Rybakowski & Jann E Schlimme & Ingrid Sibitz \\
\hline Poland & Germany & Austria \\
\hline
\end{tabular}




\begin{tabular}{|c|c|c|}
\hline Cecilia Sighinolfi & Paul St John-Smith & Taro Takeshima \\
\hline Italy & UK & Japan \\
\hline Roberta Siliquini & Wouter Staal & Qingrong Tan \\
\hline Italy & Netherlands & China \\
\hline Derrick Silove & David Stacey & Ilaria Tarricone \\
\hline Australia & Australia & Italy \\
\hline Kang Sim & Victoria Stanhope & Cumhur Tas \\
\hline Singapore & USA & Turkey \\
\hline Magenta Simmons & Jack Stevens & Alvin Kuowei Tay \\
\hline Australia & USA & Australia \\
\hline Claudia Simons & Jon Stone & David Taylor \\
\hline Netherlands & UK & UK \\
\hline Jay Singh & Martin Strassnig & Kate Tchanturia \\
\hline Norway & USA & UK \\
\hline Mark Sinyor & Barbara Stringer & Michelle Teale Sapach \\
\hline Canada & Netherlands & Canada \\
\hline Bonnie Wm Siu & Brendon Stubbs & Ghazel Tellawi \\
\hline Hong Kong & UK & USA \\
\hline Anna Smith & Paula Suárez-Pinilla & Henk Temmingh \\
\hline UK & Spain & South Africa \\
\hline Patrick Smith & Hajime Sueki & Martin Teufel \\
\hline USA & Japan & Germany \\
\hline Rebecca Smith & Koreaki Sugimoto & Michel Thibodeau \\
\hline UK & Japan & Canada \\
\hline Shubulade Smith & Sarah Sullivan & Henning Tiemeier \\
\hline UK & UK & Netherlands \\
\hline David G Smithard & Jaana Suvisaari & Nickolai Titov \\
\hline UK & Finland & Australia \\
\hline Marcel Smits & Takefumi Suzuki & Arun Tiwari \\
\hline Netherlands & Japan & Canada \\
\hline Moria Smoski & Bengt Svensson & Carmine Tomasetti \\
\hline USA & Sweden & Italy \\
\hline Anne Söderlund & Piotr Switaj & Ulysses Torres \\
\hline Sweden & Poland & Brazil \\
\hline Daya Somasundaram & Andras Szekely & Lurdes Tse \\
\hline Sri Lanka & Hungary & Canada \\
\hline Lin Sørensen & Eskinder Tafesse & Wan-Ling Tseng \\
\hline Norway & USA & USA \\
\hline Julia Friederike Sowislo & Yueh-Ming Tai & Marco Tuccori \\
\hline Switzerland & Taiwan & Italy \\
\hline Francesco Spallotta & Ricardo Taipa & James Tung \\
\hline Germany & Portugal & Canada \\
\hline Mona Srivastava & Mutsuko Takahashi & Alyna Turner \\
\hline India & Japan & Australia \\
\hline
\end{tabular}




\begin{tabular}{|c|c|c|}
\hline $\begin{array}{l}\text { Hiroyuki Uchida } \\
\text { Japan }\end{array}$ & $\begin{array}{l}\text { Marjolein Verhoeven } \\
\text { Netherlands }\end{array}$ & $\begin{array}{l}\text { Florian Weck } \\
\text { Germany }\end{array}$ \\
\hline $\begin{array}{l}\text { Alp Ucok } \\
\text { Turkey }\end{array}$ & $\begin{array}{l}\text { Jan Vevera } \\
\text { Czech Republic }\end{array}$ & $\begin{array}{l}\text { Peter M. Wehmeier } \\
\text { Germany }\end{array}$ \\
\hline $\begin{array}{l}\text { Rudolf Uher } \\
\text { Canada }\end{array}$ & $\begin{array}{l}\text { Philippe Vincent } \\
\text { Canada }\end{array}$ & $\begin{array}{l}\text { Aviv Weinstein } \\
\text { Israel }\end{array}$ \\
\hline $\begin{array}{l}\text { Zsolt Unoka } \\
\text { Hungary }\end{array}$ & $\begin{array}{l}\text { Benedetto Vitiello } \\
\text { USA }\end{array}$ & $\begin{array}{l}\text { Abraham Weizman } \\
\text { Israel }\end{array}$ \\
\hline $\begin{array}{l}\text { Dominique Valade } \\
\text { France }\end{array}$ & $\begin{array}{l}\text { David Vogel } \\
\text { USA }\end{array}$ & $\begin{array}{l}\text { David M Wellsted } \\
\text { UK }\end{array}$ \\
\hline $\begin{array}{l}\text { Tom Van Daele } \\
\text { Belgium }\end{array}$ & $\begin{array}{l}\text { Jen Vohs } \\
\text { USA }\end{array}$ & $\begin{array}{l}\text { Benedict Weobong } \\
\text { Ghana }\end{array}$ \\
\hline $\begin{array}{l}\text { Geurt Van De Glind } \\
\text { Netherlands }\end{array}$ & $\begin{array}{l}\text { Katharina Voigt } \\
\text { Germany }\end{array}$ & $\begin{array}{l}\text { Perla Werner } \\
\text { Israel }\end{array}$ \\
\hline $\begin{array}{l}\text { Fons Van De Vijver } \\
\text { Netherlands }\end{array}$ & $\begin{array}{l}\text { Corrine Voils } \\
\text { USA }\end{array}$ & $\begin{array}{l}\text { Ruud Wetzels } \\
\text { Netherlands }\end{array}$ \\
\hline $\begin{array}{l}\text { Marjan Van Den Akker } \\
\text { Netherlands }\end{array}$ & $\begin{array}{l}\text { Eberhard Voit } \\
\text { USA }\end{array}$ & $\begin{array}{l}\text { Amanda Wheeler } \\
\text { Australia }\end{array}$ \\
\hline $\begin{array}{l}\text { Rutger Jan Van Der Gaaag } \\
\text { Netherlands }\end{array}$ & $\begin{array}{l}\text { Umberto Volpe } \\
\text { Italy }\end{array}$ & $\begin{array}{l}\text { Ross White } \\
\text { UK }\end{array}$ \\
\hline $\begin{array}{l}\text { Peggy Van Der Pol } \\
\text { Netherlands }\end{array}$ & $\begin{array}{l}\text { Silke Von Esenwein } \\
\text { USA }\end{array}$ & $\begin{array}{l}\text { Richard Whittington } \\
\text { UK }\end{array}$ \\
\hline $\begin{array}{l}\text { Louk Van Der Post } \\
\text { Netherlands }\end{array}$ & $\begin{array}{l}\text { Martin Voracek } \\
\text { Austria }\end{array}$ & $\begin{array}{l}\text { Johanna Wigman } \\
\text { Netherlands }\end{array}$ \\
\hline $\begin{array}{l}\text { Annemarie Van Elburg } \\
\text { Netherlands }\end{array}$ & $\begin{array}{l}\text { Tracey Wade } \\
\text { Australia }\end{array}$ & $\begin{array}{l}\text { Dirk Wildgruber } \\
\text { Germany }\end{array}$ \\
\hline $\begin{array}{l}\text { Harm Van Marwijk } \\
\text { Netherlands }\end{array}$ & $\begin{array}{l}\text { Margda Waern } \\
\text { Sweden }\end{array}$ & $\begin{array}{l}\text { Sarah Wilker } \\
\text { Germany }\end{array}$ \\
\hline $\begin{array}{l}\text { Bregje Van Spijker } \\
\text { Australia }\end{array}$ & $\begin{array}{l}\text { Akio Wakabayashi } \\
\text { Japan }\end{array}$ & $\begin{array}{l}\text { Alishia Williams } \\
\text { Australia }\end{array}$ \\
\hline $\begin{array}{l}\text { Cornelia Van Uden-Kraan } \\
\text { Netherlands }\end{array}$ & $\begin{array}{l}\text { Fredrik A. Walby } \\
\text { Norway }\end{array}$ & $\begin{array}{l}\text { Amanda Williams } \\
\text { UK }\end{array}$ \\
\hline $\begin{array}{l}\text { Benjamin Van Voorhees } \\
\text { USA }\end{array}$ & $\begin{array}{l}\text { Wei Wang } \\
\text { China }\end{array}$ & $\begin{array}{l}\text { Monnica Williams } \\
\text { USA }\end{array}$ \\
\hline $\begin{array}{l}\text { Erik Vanderlip } \\
\text { USA }\end{array}$ & $\begin{array}{l}\text { Yuan-Pang Wang } \\
\text { Brazil }\end{array}$ & $\begin{array}{l}\text { Tony Winefield } \\
\text { Australia }\end{array}$ \\
\hline $\begin{array}{l}\text { Gustavo Vazquez } \\
\text { Argentina }\end{array}$ & $\begin{array}{l}\text { Jia Wang } \\
\text { China }\end{array}$ & $\begin{array}{l}\text { Tracy Witte } \\
\text { USA }\end{array}$ \\
\hline $\begin{array}{l}\text { Per Vendsborg } \\
\text { Denmark }\end{array}$ & $\begin{array}{l}\text { Qiang Wang } \\
\text { China }\end{array}$ & $\begin{array}{l}\text { Katrin Woitecki } \\
\text { Germany }\end{array}$ \\
\hline $\begin{array}{l}\text { Peter Ventevogel } \\
\text { Netherlands }\end{array}$ & $\begin{array}{l}\text { Norio Watanabe } \\
\text { Japan }\end{array}$ & $\begin{array}{l}\text { Owen Wolkowitz } \\
\text { USA }\end{array}$ \\
\hline $\begin{array}{l}\text { Lena Verdeli } \\
\text { USA }\end{array}$ & $\begin{array}{l}\text { Rafal Watrowski } \\
\text { Germany }\end{array}$ & $\begin{array}{l}\text { Miranda Wolpert } \\
\text { UK }\end{array}$ \\
\hline
\end{tabular}




\begin{tabular}{|c|c|c|}
\hline Eugene Wong & Barbara Yawn & Xiang Rong Zhang \\
\hline UK & USA & China \\
\hline Jesse Wright & Ksenija Yeeles & Huiping Zhang \\
\hline USA & UK & USA \\
\hline Yin Wu & Cheng-Fang Yen & Han Zhang \\
\hline UK & Taiwan & China \\
\hline Til Wykes & Yuan Yong Gui & Bin Zhang \\
\hline UK & China & China \\
\hline Brian Wymbs & Ki-Bong Yoo & Jie Zhang \\
\hline USA & Korea, South & USA \\
\hline Shifu Xiao & Nicole Yuan & Min Zhao \\
\hline China & USA & China \\
\hline Kirsten Yaffe & Kai Yuan & Jiang-Ning Zhou \\
\hline USA & China & China \\
\hline Amit Yamin & Xiaojun Yuan & Gang Zhu \\
\hline Israel & USA & China \\
\hline Chih Chieh Yang & David Yusko & Tim Ziermans \\
\hline USA & USA & Netherlands \\
\hline Yen Kuang Yang & Clement Zai & Stephan Zipfel \\
\hline Taiwan & Canada & Germany \\
\hline Philip Yanos & Yu-Feng Zang & Phillip Zoladz \\
\hline USA & China & USA \\
\hline Shuqiao Yao & Faika Zanjani & Jon-Kar Zubieta \\
\hline China & USA & USA \\
\hline Norio Yasui-Furukori & Yuliya Zaytseva & Maria Victoria Zunzunegui \\
\hline Japan & Germany & Canada \\
\hline Wai-Ying Wendy Yau & Patrizia Zeppegno & \\
\hline USA & Italy & \\
\hline
\end{tabular}

